\title{
Prolificidade de caprinos mestiços leiteiros no semiárido nordestino
}

\section{José Lindenberg Rocha Sarmento ${ }^{1,5}$, Edgard Cavalcanti Pimenta Filho 2,5 , Urbano Gomes Pinto de Abreu $^{3}$, Maria Norma Ribeiro ${ }^{4,5}$, José Ernandes Rufino de Sousa ${ }^{1}$}

1 UFPI/Campus de Bom Jesus-PI, CEP: 64.900-000

2 DZ/UFPB.

3 Embrapa Pantanal.

${ }^{4}$ DZ/UFRPE.

${ }^{5}$ Bolsista do CNPq

RESUMO - Utilizaram-se 526 registros de parições de cabras mestiças com o objetivo de avaliar a influência de alguns efeitos de ambiente sobre a prolificidade. As análises estatísticas foram realizadas por meio de modelos lineares generalizados, empregando-se a função de distribuição binomial e a função de ligação logística. As probabilidades de ocorrência de partos múltiplos foram calculadas por meio de um modelo que incluiu os efeitos de ano e mês de cobertura, ordem de parto, idade ao primeiro parto, peso à cobertura, produção de leite, duração da lactação e intervalo de partos. A prolificidade média do rebanho foi de 1,49 cabrito. Os efeitos de mês de cobertura, ordem de parto, peso na cobertura, idade ao primeiro parto e intervalo de partos foram significativos. As chances de partos múltiplos tenderam a aumentar com o avanço da idade da fêmea (ordem) e do peso à cobrição. Fêmeas cobertas no período de disponibilidade de alimentos apresentaram maior probabilidade de partos múltiplos. A idade ao primeiro parto foi outra variável importante, pois fêmeas com maior idade apresentaram maior probabilidade de partos múltiplos. As fêmeas com maior intervalo de parto tenderam a apresentar maiores probabilidades de parir mais de uma cria. A prolificidade de caprinos mestiços é altamente afetada pela variação de ambiente, maturidade fisiológica, condição corporal e idade de início da vida reprodutiva.

Palavras-chave: efeitos de ambiente, eficiência reprodutiva, modelos lineares generalizados, ordem de parto, peso à cobertura

\section{Litter size of crossbreed dairy goats in the northeastern semi-arid}

ABSTRACT - The aim of this study was to evaluate the influence of some environmental effects on the litter size, using 526 records of kidding of dairy crossbreed goats. The statistical analyses were carried out through generalized linear models, using a binomial distribution function and the logistic link function. The probabilities of occurrence of multiple kidding were calculated by a model that included the effects of year and month of mating, order of kidding, age at first kidding, weight at mating, milk yield, lactation length, and kidding interval. The average litter size of the herd was 1.49 kids. The effects of month of mating, order of kidding, weight at mating, age at first kidding, and kidding interval were significant. Chances of multiple kiddings tended to increase with the age of dam (order) and weight at mating. Females mated during food availability period presented larger probabilities of multiple births. The age at first kidding was another important variable because females with higher age presented higher probability of multiple kiddings. Females with larger kidding intervals tended to present higher probabilities of kidding more than one kid. The litter size of crossbreed goats is highly affected by environmental changes, physiologic maturity (age), body condition and age at beginning of the reproductive life.

Key Words: environment effects, generalized linear models, mating weight, order of kidding, reproductive efficiency

\section{Introdução}

A eficiência de um sistema de produção animal é determinada por eventos produtivos e reprodutivos inerentes às fêmeas, bem como ao desenvolvimento ponderal de suas proles. Entre as características reprodutivas, a prolificidade (número de cabritos nascido por fêmea parida) é uma das mais importantes para determinação da eficiência do sistema, pois está diretamente relacionada com a viabilidade econômica da exploração do rebanho. Para Danell (1986), o valor econômico relativo do aumento de uma cria, no número de crias nascidas por ovelha, foi estimado ser 16,4 vezes maior do que o aumento de $1,0 \mathrm{~kg}$ no peso da cria aos 120 dias de idade, o que sugere a importância desta característica para os caprinos. 
A prolificidade contribui sobremaneira com o ganho genético anual nos rebanhos em que se pratica seleção, uma vez que menores índices de prolificidade implicam menor número de cabritos nascidos por ano, o que dificulta a reposição do plantel, diminui a pressão de seleção e aumenta o intervalo de geração, além de diminuir a taxa de desfrute do rebanho. Além disso, esta característica é de fácil mensuração e, de acordo com Rao \& Notter (2000), possui herdabilidade alta, quando comparada a outras características reprodutivas, o que sugere rápida resposta à seleção. Todavia, o melhoramento para prolificidade pode promover efeito adverso sobre a produção global do rebanho e, consequentemente, reduzir o desempenho econômico do rebanho, devido às relações antagônicas encontradas entre características de importância econômica, como verificado por Subandryo (1994) e Menendez-Buxadera et al. (2003).

Devido à sua importância, deve-se procurar identificar os fatores que interferem sobre a característica, de modo a subsidiar a maximização do número de crias durante a vida reprodutiva da fêmea. A literatura nacional ainda é incipiente no que diz respeito a estudos de algumas características reprodutivas de caprinos. Os escassos artigos verificados na literatura nacional (Silva \& Mello, 1996; e Santos et al., 1998) buscaram avaliar a influência de alguns efeitos do ambiente sobre a referida característica e empregaram modelos que pressupõem distribuição normal, quando, na prática, isso não ocorre, uma vez que esta tem distribuição discreta.

Desta forma, objetivou-se avaliar alguns efeitos do ambiente que podem interferir na prolificidade de um rebanho caprino leiteiro, empregando a teoria de modelos lineares generalizados.

\section{Material e Métodos}

O trabalho foi realizado com o rebanho caprino da Fazenda Carnaúba, pertencente à AMDA (Agropecuária Manoel Dantas Ltda.), situada no município de Taperoá, microrregião do Cariri Ocidental da Paraíba. Os registros de prolificidade foram obtidos a partir do controle reprodutivo das cabras mestiças oriundas do cruzamento de reprodutores da raça Pardo Alpino com fêmeas do tipo nativo Gurguéia, coletados de 1990 a 1998. Inicialmente, o arquivo continha 1.939 registros. Para gerar o arquivo utilizado nas análises, foram feitas algumas restrições, permanecendo no arquivo cabras com pai e mãe conhecidos, bodes com, no mínimo, cinco filhas no rebanho e cabras com, no máximo, sete parições, restando 518 registros para as análises.

Os animais foram criados em sistema semiextensivo e alimentados com capim-buffel (Cenchrus ciliaris L.) e pasto nativo, na estação chuvosa, e capim-elefante (Pennisetum purpureum schum.), palma forrageira (Opuntia sp.), raspa de mandioca (Manihot sativa), bagaço de cana hidrolisado (Sacharum oficcinarum) e concentrado proteico, na estação seca, além de sal mineral à vontade. Utilizou-se o sistema de monta natural controlada e controle sanitário, sistematicamente. A partir das fichas de produção e reprodução, foi editado um arquivo contendo o número da cabra, paternidade e maternidade, data de nascimento, datas dos partos, produção de leite, duração da lactação, tipo, ordem e intervalo de partos.

Os dados de prolificidade estudados - parto simples ou múltiplo (dois ou mais), são provenientes de uma distribuição discreta e, neste caso, com duas classes, com uma probabilidade de ocorrência de cada classe, simples ou múltiplo. Desta forma, a análise desse tipo de informação objetiva modelar a probabilidade de ocorrência de cada classe em função de variáveis explicativas (efeitos de ambiente). Recentemente, novas metodologias têm sido desenvolvidas para esse fim. Segundo Abreu et al. (2006), as equações de estimação generalizadas, uma extensão do modelo linear generalizado, fornecem uma abordagem semiparamétrica na análise de dados univariados, em que o método de estimação permite a modelagem de variáveis respostas com a distribuição de interesse.

Para análise estatística da característica em estudo, utilizou-se o procedimento GENMOD contido no programa SAS (1999). O GENMOD ajusta modelos lineares generalizados, que são amplamente robustos, pelo método da máxima verossimilhança, utilizando uma função de ligação apropriada à probabilidade de distribuição. Utilizou-se a função de distribuição binomial e a função de ligação logística. O teste de qui-quadrado $\left(\chi^{2}\right)$ de Pearson foi utilizado para verificar o ajuste da função aos dados.

Dentro do procedimento GENMOD, utilizou-se a opção ESTIMATE, que calcula o logaritmo da razão de chances, $\ln \left[P_{i} / 1-P_{i}\right]$, em que $P_{\mathrm{i}}$ representa a probabilidade de o parto ser múltiplo e $1-\mathrm{P}_{\mathrm{i}}$ a probabilidade de ser simples. Para que os coeficientes estimados refletissem o efeito de o parto ser múltiplo, em relação ao simples, calculou-se a exponencial do logaritmo da razão de chances, por meio da opção “EXP”, também disponível neste procedimento mencionado, cuja resposta representa o número de vezes que a classe de parto múltiplo teria mais chances de ocorrer que a classe de parto simples.

As probabilidades de ocorrência de partos múltiplos foram calculadas como se segue: $\mathrm{P}_{\mathrm{i}}=\mathrm{e} / 1+\mathrm{e}$, em que: $\mathrm{e}=\mu+\mathrm{AC}+\mathrm{MC}+\mathrm{OP}+\sum_{\mathrm{i}=1}^{2} \beta_{\mathrm{i}}(\mathrm{IPP})^{i}+\sum_{\mathrm{i}=1}^{2} \alpha_{\mathrm{i}}(\mathrm{PC})^{\mathrm{j}}, \quad$ em que

R. Bras. Zootec., v.39, n.7, p.1471-1476, 2010 
$\mu=$ constante inerente a todas as observações; AC = efeito do ano de cobertura; $\mathrm{MC}$ = efeito do mês de cobertura; $\mathrm{OP}=$ efeito da ordem de parto (variando de um a sete); $\beta_{i}=$ coeficientes de regressão da idade ao primeiro parto (linear e quadrático); $\alpha_{i}=$ coeficientes de regressão do peso da fêmea no momento da cobertura (linear e quadrático). A significância dos efeitos considerados foi inicialmente testada pela estatística da razão de verossimilhança a 5\% de probabilidade.

Posteriormente, as probabilidades de ocorrência de partos múltiplos foram calculadas mantendo-se os efeitos significativos da análise anterior e incluindo-se a produção de leite, a duração da lactação e o intervalo de parto como covariáveis linear e quadrática, referentes ao parto anterior, objetivando avaliar a relação destas características com a prolificidade. Na segunda fase das análises, o primeiro parto foi suprimido da análise por não haver, naturalmente, a influência de partos anteriores.

\section{Resultados e Discussão}

A função de distribuição binomial e a função de ligação logística apresentaram ajuste adequado, como sugerido pelo $\chi^{2}$ de Pearson. A prolificidade média do rebanho foi de 1,35 cabrito por fêmea, com coeficiente de variação igual a 35\%. A frequência de ocorrência de partos simples e múltiplos no rebanho foi, respectivamente, $64,5 \%$ e $35,5 \%$. Médias superiores a estas foram relatadas por Silva \& Araújo (2000) ao estudarem caprinos mestiços oriundos de cruzamentos entre as raças Alpina, Moxotó e Anglo-Nubiana.

As análises estatísticas revelaram que os efeitos do mês de cobertura, ordem de parto e o efeito linear do peso na cobertura e da idade no primeiro parto foram significativos $(\mathrm{P}<0,05)$, indicando que esses efeitos devem ser considerados no estudo desta característica. Santos et al. (1998), utilizando informações de prolificidade deste mesmo rebanho, porém empregando a metodologia de quadrados mínimos ordinários, encontraram efeito significativo apenas da ordem de parto. Silva \& Araújo (2000), estudando um rebanho de cabras mestiças no Brasil, porém utilizando a metodologia de quadrados mínimos, verificaram efeito significativo apenas para o ano de parição; já os efeitos de grupo genético, ordem de parto e peso da mãe no parto não foram importantes fontes de variação. Amoah et al. (1996) verificaram diferenças de prolificidade entre várias raças estudadas, todavia os autores destacaram o peso na cobrição, onde a análise regressão linear revelou um aumento de 0,03 cabrito por kg de peso na cobrição.
As chances de ocorrência de partos múltiplos (Tabela 1) tenderam a aumentar com o aumento da idade da fêmea (ordem do parto); ou seja, à medida que a fêmea tornou-se anatômica e fisiologicamente mais preparada. As maiores diferenças observadas foram em relação às chances estimadas entre o primeiro e os demais partos (Tabela 1), o que sugere menor condição fisiológica das fêmeas na primeira cobertura. Fêmeas de segunda parição apresentaram 3,75 vezes mais chances de ter parto múltiplo que as de primeira; as de terceira 6,34 vezes mais chances que a de primeira e assim sucessivamente, até as de sétima parição, que apresentaram 26,4 vezes mais chances de parirem duas ou mais crias. As diferenças da segunda ordem, em relação às superiores, foram menores em comparação às de primeira. As chances de ocorrência de partos múltiplos foram próximas entre as fêmeas com três ou mais partos, uma vez que as fêmeas nesse estágio já atingiram a maturidade fisiológica. As fêmeas com sete partos, como exceção, apresentaram chances significativamente maiores que as de terceira e quinta ordem. Silva \& Araújo (2000), estudando caprinos mestiços no Nordeste do Brasil por meio de uma metodologia que pressupõe normalidade, verificaram influência da ordem do parto sobre a prolificidade. Estes autores verificaram uma variação do primeiro ao quinto de 1,47 a 1,82 cabrito/parto e atribuíram essa variação ao estado fisiológico da matriz, principalmente do aparelho reprodutor.

O comportamento verificado nas primíparas neste estudo parece biologicamente correto, uma vez que fêmeas em idades mais jovens não têm alcançado a maturidade necessária do sistema reprodutor, em termos anatômicos e fisiológicos, refletindo maior frequência de partos simples. Efeitos significativos da ordem do parto sobre a prolificidade foram encontrados por Santos et al. (1998). Diferindo dos resultados obtidos neste estudo, Amoah et al. (1996) e Silva \& Mello (1996) relataram que este efeito não foi importante fonte de variação sobre a prolificidade. Vale ressaltar que, em ambos os trabalhos citados, a metodologia utilizada pressupunha distribuição normal.

Tabela 1 - Razão de chances para a ocorrência de partos múltiplos de fêmeas com diferentes ordens de parição

\begin{tabular}{ccccccc}
\hline Ordem de parto & 1 & 2 & 3 & 4 & 5 & 6 \\
\hline 2 & $3,3^{*}$ & & & & & \\
3 & $5,2^{*}$ & $1,6 \mathrm{~ns}$ & & & & \\
4 & $9,4^{*}$ & $2,8^{*}$ & $1,8 \mathrm{~ns}$ & & & \\
5 & $4,5^{*}$ & $1,4 \mathrm{~ns}$ & $0,8 \mathrm{~ns}$ & $0,5 \mathrm{~ns}$ & & \\
6 & $7,6^{*}$ & $2,3 \mathrm{~ns}$ & $1,5 \mathrm{~ns}$ & $0,8 \mathrm{~ns}$ & $1,7 \mathrm{~ns}$ & \\
7 & $14,7^{*}$ & $4,5^{*}$ & $2,8 \mathrm{~ns}$ & $1,6 \mathrm{~ns} 3,3 \mathrm{~ns}$ & $1,9 \mathrm{~ns}$ \\
\hline
\end{tabular}

* Significativo a 5\% de probabilidade pela estatística da razão de verossimilhança; ns não-significativo. 
Como a metodologia utilizada permite calcular a probabilidade de ocorrência de partos múltiplos, considerando os efeitos significativos incluídos no modelo de análise, avaliou-se o comportamento das probabilidades em função da ordem do parto, do peso na cobertura, mês de cobertura e da idade no primeiro parto. Para ilustrar melhor, as comparações foram feitas considerando dois meses (chuvoso, março, e seco, novembro), três situações de idade no primeiro parto (baixa, média e alta) em função do peso na cobertura, para cada ordem de parto (Figura 1). Verificou-se aumento considerável na probabilidade de parições múltiplas com o aumento do peso na cobrição e da
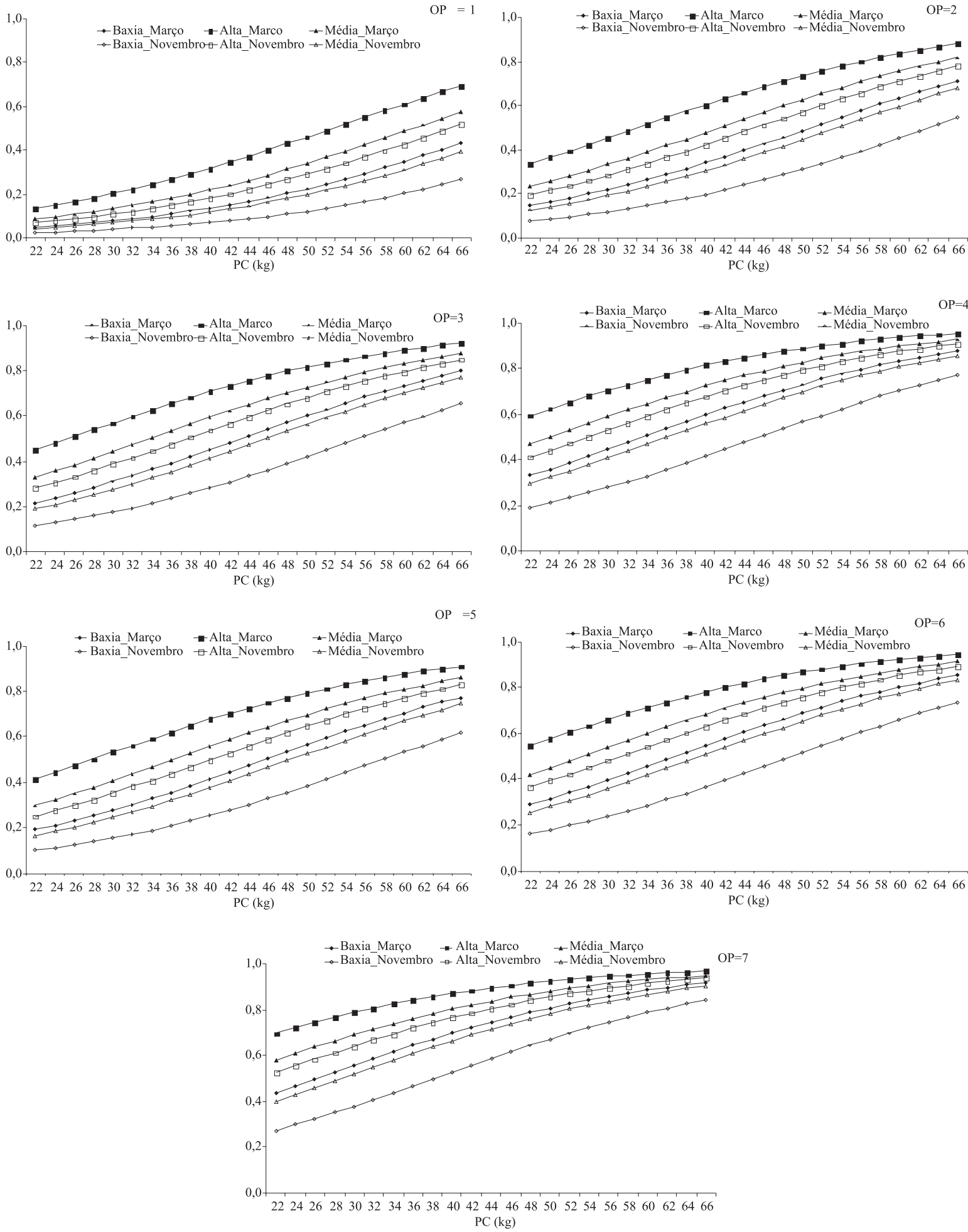

Figura 1 - Probabilidades de ocorrência de partos múltiplos em função da ordem de parição (OP), mês de cobertura (março e novembro), idade no primeiro parto (baixa, média e alta) e peso na cobertura (PC). 
ordem de partos (idade). Fêmeas leves e no primeiro parto apresentaram baixa probabilidade de parir mais de uma cria (inferior a 0,1), embora esta tenha aumentado de acordo com o peso na cobrição da fêmea, o que evidencia a importância da condição corporal no momento da concepção sobre o número de óvulos fecundados ou viabilizados em fetos. Por outro lado, fêmeas pesadas e com sete parições, o que caracteriza boa condição corporal e maturidade fisiológica, apresentaram probabilidade de ter partos múltiplos superior a 0,95 (Figura 1). O resultado verificado em relação ao peso na cobrição está de acordo com verificado por Amoah et al. (1996), os quais verificaram efeito do peso na cobertura sobre a prolificidade em várias raças caprinas, mesmo empregando um modelo linear que pressupõe normalidade dos dados.

Com relação ao efeito de mês de cobrição, ficou evidente a influência deste sobre a prolificidade. Fêmeas cobertas no período de maior disponibilidade de alimentos (março) apresentaram probabilidades maiores de proporcionarem nascimentos múltiplos do que as cobertas no mês de novembro, independente da idade no primeiro parto e da ordem de partos (Figura 1). Possivelmente, este resultado se justifica pelo maior nível nutricional da fêmea no momento da cobertura, oriundo da maior disponibilidade de forragem no período da chuva. Além disso, esses resultados sugerem que a habilidade de proporcionar melhor condição corporal ou peso vivo na época dos acasalamentos melhora a taxa de ovulação, o que ocasiona aumento na prolificidade. Os trabalhos consultados na literatura não apontaram para a importância deste efeito.

A idade no primeiro parto foi outra variável importante na expressão da prolificidade para o rebanho em estudo. Fêmeas com maior idade no primeiro parto apresentaram maior probabilidade de partos múltiplos, diferentemente daquelas de baixa idade no primeiro parto, as quais apresentaram maior probabilidade de parir uma única cria. Esse comportamento foi verificado em todas as ordens de parto e independente do peso na cobrição (Figura 1). Na situação de primeiro parto e com fêmeas leves, as probabilidades de partos múltiplos foram baixas, embora quando a idade aumentou tenha se observado ligeira tendência de aumento da probabilidade. Estes resultados são condizentes com a realidade do rebanho em estudo, dado que não foi imposto às fêmeas idade mínima para que fossem cobertas pela primeira vez. Possivelmente, este fato foi o motivo principal para que o efeito da idade no primeiro parto tenha se prolongado até a sétima ordem, embora as diferenças de probabilidades tendessem a diminuir nesta última ordem de parição.
Ao analisar as características produção de leite, duração da lactação e intervalo de parto, referente ao parto anterior, verificou-se que apenas o intervalo de partos mostrou-se significativo sobre a prolificidade $(\mathrm{P}<0,05)$, com efeito quadrático. Fêmeas com maiores intervalos de parto tenderam a apresentar maiores probabilidades de parirem mais de uma cria (Figura 2). Estas probabilidades aumentaram com o aumento em peso, como mostrado anteriormente, e com o aumento de idade (OP) das fêmeas, ou seja, fêmeas
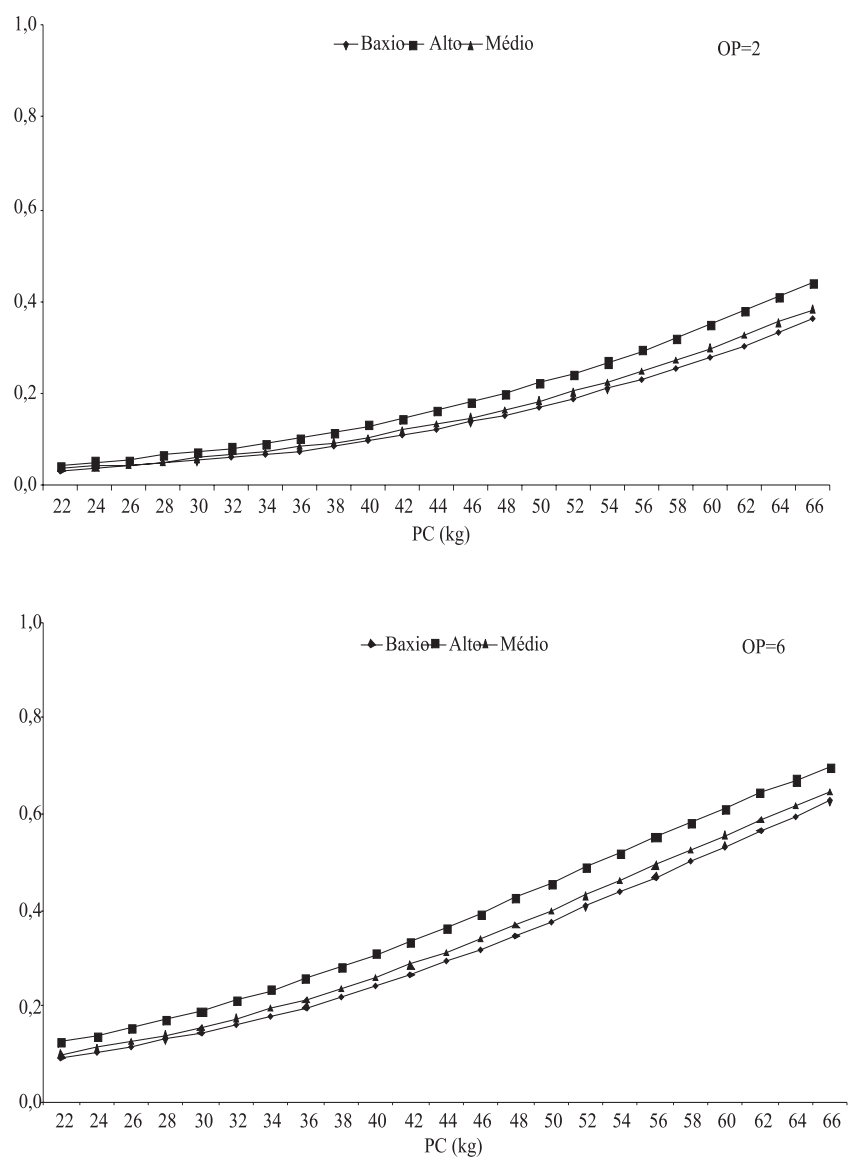

Figura 2 - Probabilidade de ocorrência de partos múltiplos em função da ordem de parição (OP), do intervalo de parto (baixo, médio e alto) e do peso na cobertura (PC).

com seis parições apresentaram maior probabilidade de partos múltiplos que as com dois partos. Esse comportamento parece correto, uma vez que fêmeas com menores intervalos de parto podem ter maior desgaste do sistema reprodutivo por não ter um período de tempo suficiente para se recompor para o próximo parto, refletindo em menor taxa de ovulação e, consequentemente, menor possibilidade de partos múltiplos. 


\section{Conclusões}

A prolificidade é afetada pela variação de ambiente (mês), pela maturidade fisiológica (ordem de parição), condição corporal (peso) e pela idade de início da vida reprodutiva da fêmea. Melhorias nessa característica podem ser mais facilmente obtidas com ajuste no manejo alimentar e adequação da idade mínima para o início da vida reprodutiva.

\section{Agradecimentos}

Ao Conselho Nacional de Desenvolvimento Científico e Tecnológico (CNPq), pelo financiamento da pesquisa, e à Agropecuária Manoel Dantas Ltda (AMDA), pela cessão dos dados.

\section{Referências}

ABREU, U.G.P.; LOPES, P.S.; TORRES, R.A. et al. Avaliação da introdução de tecnologias no sistema de produção de gado de corte no Pantanal. Desempenho e descarte de matrizes. Revista Brasileira de Zootecnia, v.35, n.6, p.2496-2503, 2006.
AMOAH, E.A.; GELAYE, S.; GUTHRIE, P. et al. Breeding season and aspects of reproduction of female goats. Journal of Animal Science, v.74, p.723-728, 1996.

DANELL, O. Selection index for pelt lambs. Uppsala: Swedish University Agriculture Science, Department Animal Breeding \& Genetics, 1986 (Publication, 9).

MENÉNDEZ-BUXADERA, A.; ALEXANDRE, G.; MANDONNET, N. et al. Direct genetic and maternal effects affecting litter size, Barth weight and pre-weaning losses in Creole goats of Guadeloupe. Animal Science, v.77, p.363-36, 2003.

SANTOS, E. M.; PIMENTA FILHO, E. C.; RIBEIRO, M. N. et al. Estudo das variáveis que afetam a prolificidade de um rebanho caprino leiteiro no semi-árido paraibano. In: REUNIÃO ANUAL DA SOCIEDADE BRASILEIRA PARA O PROGRESSO DA CIÊNCIA, 50., 1998, Natal. Anais... Natal: SBPC, 1998 (CD-ROM).

SILVA, F.L.R.; MELLO, A.A. Produção de leite e prolificidade em cabras mestiças no Semi-Àrido. In: REUNIÃO ANUAL DA SOCIEDADE BRASILEIRA DE ZOOTECNIA, 33., 1996, Fotaleza. Anais... Fortaleza: SBZ, 1996 (CD-ROM).

SILVA, F.L.R.; ARAÚJO, A.M. Desempenho produtivo em caprinos mestiços no semi-árido do nordeste do Brasil. Revista Brasileira de Zootecnia, v.29, n.4, p.1028-1035, 2000.

STATISTICAL ANALYSIS SYSTEM - SAS. User's guide: statistics. Version 8.0. Cary: SAS Institute, 1999. p.1364-1462.

SUBANDRIYO, I.I. Genetic and environmental factors affecting birth weight, weaning weight and preweaning survial rates of prolific javanese sheep. In: WORLD CONGRESS ON GENETICS APPLIED TO LIVESTOCK PRODUCTION, 5., 1994, Guelph. Proceedings... Guelph, 1994. v.18, p.127-130.

RAO, S.; NOTTER, D.R. Genetic analysis of litter size in Targhee, Suffolk and Polypay sheep. Journal of Animal Science, v.78, p.2113-2120, 2000. 